\title{
Evolutionary dynamics of human autoimmune disease genes and malfunctioned immunological genes
}

\author{
Soumita Podder and Tapash Chandra Ghosh ${ }^{*}$
}

\begin{abstract}
Background: One of the main issues of molecular evolution is to divulge the principles in dictating the evolutionary rate differences among various gene classes. Immunological genes have received considerable attention in evolutionary biology as candidates for local adaptation and for studying functionally important polymorphisms. The normal structure and function of immunological genes will be distorted when they experience mutations leading to immunological dysfunctions.

Results: Here, we examined the fundamental differences between the genes which on mutation give rise to autoimmune or other immune system related diseases and the immunological genes that do not cause any disease phenotypes. Although the disease genes examined are analogous to non-disease genes in product, expression, function, and pathway affiliation, a statistically significant decrease in evolutionary rate has been found in autoimmune disease genes relative to all other immune related diseases and non-disease genes. Possible ways of accumulation of mutation in the three steps of the central dogma (DNA-mRNA-Protein) have been studied to trace the mutational effects predisposed to disease consequence and acquiring higher selection pressure. Principal Component Analysis and Multivariate Regression Analysis have established the predominant role of single nucleotide polymorphisms in guiding the evolutionary rate of immunological disease and non-disease genes followed by m-RNA abundance, paralogs number, fraction of phosphorylation residue, alternatively spliced exon, protein residue burial and protein disorder.

Conclusions: Our study provides an empirical insight into the etiology of autoimmune disease genes and other immunological diseases. The immediate utility of our study is to help in disease gene identification and may also help in medicinal improvement of immune related disease.
\end{abstract}

Keywords: Autoimmune disease, Immunological genes, Evolutionary rate, SNPs, Alternative splicing

\section{Background}

The knowledge gleaned from several in silico studies has facilitated in understanding the variability of evolutionary patterns in gene classes that can illuminate their inherent characteristics. In particular, studies on the functional and evolutionary attributes of human immune system have attained a major focus since it is an orchestra of various defense mechanisms whereby human body maintains functional and organizational integrity against foreign encroachment. The evolutionary

\footnotetext{
* Correspondence: tapash@boseinst.ernet.in

Bioinformatics Centre, Bose Institute, P 1/12, C.I.T. Scheme VII M, Kolkata 700 054, India

history of insects, chicken and mammals indicates that the majority of immune response genes are subjected to positive selection than remainder of the genes [1-3]. Immune response genes are also found to exhibit rapid gene turn over i.e. gene gain and loss [4]. Contextually, it has been proposed that probability of disease predisposition is higher in the genes with high rates of non synonymous mutations [5]. Diseases caused by abnormal or absences of immunologic mechanisms are thus very much common. According to disease mechanism, immune system linked disease genes are generally categorized into two broad classes (i) Immunodeficiencydysregulation of the immune system in eliminating 
microbial antigens resulting in chronic immunologic inactivation predisposed to immunologic disorder such as AIDS, DiGeorge syndrome, Chronic granulomatous disease, Wiskott-Aldrich Syndrome, Hypersensitivity etc [6]. (ii) Autoimmunity- mistakenly immune system launches attacks on its own tissue by confusing itself as a foreign invader, leading to autoimmune disorder e.g. Graves disease, Rheumatoid arthritis, Multiple sclerosis, Goodpasture's syndrome etc [6]. Till date various analyses with autoimmune diseases have attempted to figure out their novel characteristics and possible mechanisms [7-10]. Recently, it has been hypothesized that some evolutionarily conserved proteins, present in pathogenic, commensal organisms and their hosts, provide the stimulus that initiates autoimmune disease in susceptible individuals [9]. A possible mechanism of autoantigen formation was thought to be instigated by increased non-canonical splicing that renders untolerized epitopes on antigen [10]. Although disorders caused by dysregulation of immune system have been studied in separate disease class, unique features of that entire disease genes class are still uncharacterized.

Recently, more extensive focus has been concentrated to scrutinize the disease genes for their unique characteristics that distinguish them from the remainder of the genome [11-13]. The growing incidence of autoimmune and several immunological diseases have prompted us to delve into the genic or proteomic features which induce the disease causing mutation on host defense genes. Evaluating the properties of functional immunological genes, malfunctioned immunological genes and autoimmune disease genes in the evolutionary framework we postulate that the autoimmune disease genes are under the strongest purifying selection among the three classes. We exemplified the underlying reasons by assessing the mutational effect at DNA-mRNA-protein levels. The comprehensive cataloguing and characterization of genes from evolutionary perspective may provide the basis for determining how nucleotide substitution impacts biological function and instigate common human diseases. Identification of the various features that are responsible to distinguish between several immunological disease and non-disease genes may help to identify the probable biochemical basis for the disease incidence. Our work may be extended in future in the form of refining the specialized features of functional and disease causing immunological genes.

\section{Results}

Evolutionary Dynamics of Immune Related Disease and Non-disease Genes

The available resources of immunological disease genes facilitate us to investigate the evolutionary pressure acting on the autoimmune disease genes (AD) and other classes of disease genes resulting from malfunctioning immunological genes (ID) with respect to the immunological genes (IG) without known association to disease. Our result depicts a significant $\left[P\right.$ value $=7 \times 10^{-3}(\mathrm{AD}$ vs ID); $1 \times 10^{-3}$ (ID vs IG); $4.6 \times 10^{-2}$ (AD vs IG)] gradual increase in the ratio of non-synonymous to synonymous substitutions $(\omega)$ [AD (mean $\omega=0.336$ ); ID (mean $\omega=0.344$ ); IG (mean $\omega=0.446)]$. Such evolutionary dynamics of disease and non-disease genes linked to immunological genes is a bit surprising because disease genes intuitively experience more mutational changes than non-disease genes, yet they are unable to escape the evolutionary pressure. It is obvious that mutation or variation will occur either in gene or m-RNA or protein level or in all the three levels to confer disease phenotypes. We intend to investigate in which level the mutation persuade the persistence of selection pressure.

\section{Effects of Gene level variations}

The first wave of information from the human genome analysis has revealed that single nucleotide polymorphisms (SNPs) is the major resource of genetic and phenotypic variations in human. Scanning for the signatures of positive selection in human population suggests that SNPs in protein coding regions show regional evidence of less intense purifying selection $[14,15]$. Investigating the impact of SNPs in the coding region of above gene classes exemplified that accumulation of non-synonymous SNPs is significantly higher $(\mathrm{Z}$ score $=2.37$, confidence level $=$ 95\%) in case of the AD (78.36\%) compared to the ID (73.04\%) genes. Moreover, IG genes are themselves less prone to non-synonymous substitutions $(69.05 \%)$ than both classes of disease genes [AD Vs IG $(\mathrm{Z}$ score $=5.0125$ confidence level $=95 \%$ ); ID Vs IG ( $Z$ score $=2.011$ confidence level $=95 \%)$ ]. This observation clearly depicts that the most conserved group of genes is indeed the most sensitive ones to variation.

Secondly, the shuffling of genes brought about by genetic recombination is a major engine of genetic variation. Recombination rate (RR) has been found to have a positive correlation with DNA diversity in many organisms, both in animals [16-18] and in plants [19]. Thus, accumulation of higher amount of SNPs was expected to initiate the higher RR for AD compared to ID and IG and the result was also in accordance to the expectation (average $\mathrm{RR}(\mathrm{cM} / \mathrm{Mb})$ for $\mathrm{AD}=0.051, \mathrm{ID}=0.035, \mathrm{IG}=0.0023$; each value is significant at least at $P<0.05$ level in MannWhitney test). Though the mutagenic nature of recombination rate may reflect the possibility of higher non-synonymous substitutions, the prevalence of Hill-Robertson interference in the genomic regions with higher RR have been proposed to increase the efficacy of purifying selection $[20,21]$. Moreover, a positive association has been 
asserted between RR and gene expression level which also explains the lower evolutionary rate in regions with higher recombination frequencies [22]. Analyzing microarray expression data we also observed that on average the $\mathrm{AD}$ genes tend to be more highly expressed than the other two classes of genes (average expression for $\mathrm{AD}=238.266$; ID $=175.138 ;$ IG $=128.497$; each value is significant at least at $P<0.05$ level in Mann-Whitney test). In addition to that, RR has long been thought to be one of the principal forces behind the gene duplication frequency [23,24]. Calculating the paralogs number in three groups of genes emphasized that the AD genes acquired a large number of duplicates compared to ID and IG genes (Average paralogs per genes for $\mathrm{AD}=10.006$; $\mathrm{ID}=8.61, \mathrm{IG}=6.32$; each value was significant at least at $P<0.05$ level in MannWhitney test). Higher duplicability may enforce the slower evolutionary rate on AD genes in contrast to other two classes since duplicated genes encounter more purifying selection than singletons even though shortly after the duplication, they experience a considerable relaxation of selection pressure [25]. From this it can be inferred that the SNPs and recombination rate collectively incite recurrent gene duplication (Spearman's $\rho_{\mathrm{SNP}, \mathrm{RR}}=0.120, \mathrm{P}=$ $1.0 \times 10^{-3}$; Spearman's $\rho_{R R}$, paralogs number $=0.060, \mathrm{P}=1.0$ $\times 10^{-3}$ ) and elicit the selection pressure on disease genes.

With the advent of genome scanning technology it has uncovered that the human genome becomes structurally dynamic due to the presence of thousands of heritable copy of mutation and are equally important as SNPs [26]. It was reported that reduced purifying selection has been acting upon copy number variants (CNVs) region [27]. Looking for the association of CNVs with immunological disease and non-disease genes we noticed that the nondisease immunological genes are significantly (Z-value $=$ 1.96 at $95 \%$ confidence level) more prone (53.98\% of total immunological genes) to suffer from CNVs compared to other immunological disease genes $(49.72 \%$ of total other immunological disease genes) while the later group of disease genes ( $44.5 \%$ of total autoimmune disease genes) exhibit significantly ( $Z$ value $=1.99$ at $95 \%$ confidence level) lesser CNVs than other immunological disease genes. These findings are also consistent with the notion that the CNV genes prefer to encode large numbers of secreted, olfactory, and immunity proteins rather than the genes harboring Mendelian disease [28]. Although the disease genes concerned in our study are inherited by both Mendelian and non-Mendelian fashion, we did not observe any opposite trend for accounting the non-Mendelian disease genes.

\section{Effects of Transcript level Variations}

Over the past decade, it has been postulated that alternative splicing (AS) is a critical post transcriptional event directing an enhancement of transcriptome and proteome diversity, particularly in higher organisms [29]. The frequent accumulation of non-synonymous mutations in alternatively spliced regions [30] initiates a faster rate of evolution in alternatively spliced exons than the constitutively spliced ones as evidenced from a comparison of orthologous human and mouse genes [31]. Investigation on the involvement of the three groups of genes in alternative splicing mechanism revealed that most of the IG genes favor alternative splicing to increase their proteomic diversity in contrast to AD and ID genes (Table 1). Accordingly, the profuse number of alternatively spliced exons are encompassed in IG genes compared to ID and AD genes (average alternatively spliced exons per gene in $\mathrm{AD}=5.89$, ID = $6.78, \mathrm{IG}=8.85$; each value is significant at least at $P<0.05$ level in Mann-Whitney test). Such nature of IG is also biologically relevant since it was proposed that AS is crucial for a functional immune system as it offers the potency of high degree of diversity and the competence of individual cells to rapidly adapt and respond towards the changing environmental conditions [32,33].

Since, alternative splicing can bolster organism complexity by effectively increasing the proteome size, the m-RNA abundance would be higher for the immunological genes. However, we already noticed IG genes are lowly expressed. Accounting EST data, the trend remain exactly same i.e. the EST count/m-RNA abundance is lower for the IG (27.02) compared to ID (35.11) and AD (48.72) genes. Hence, we ask what drives the lower mRNA abundance of AS rich immunological genes. In the recent year it has been clarified that up to one-third of human AS events create a premature termination codon (PTC) that would cause the resulting mRNA to be degraded by nonsense-mediated mRNA decay (NMD) $[34,35]$ and it was also stated that a higher rate of mRNA decay can be considered as an indicator of the lower gene expressivity [36]. Analysis on the coupling of NMD to the AS linked genes shows that most of the alternatively spliced isoforms of IG undergo mRNA decay while the count is much lower for ID and AD genes (Table 1).

Another implication of alternative splicing is to promote intrinsically disordered protein, thus enabling functional and regulatory diversity in human proteome $[37,38]$. Calculation of disorder residues in the three classes of proteins shows that the percentage of unstructured protein regions in IG, ID and AD genes are respectively $44.23 \%, 32.22 \%$ and $21.52 \%$ and the difference between each of the above values is significant at $\mathrm{P}$ $<0.05$ (in Mann-Whitney test). The aberrant increase of disorderness in IG proteins again confirms the high flexibility of antigen binding sites in immunoglobulin

to combat against an almost infinite diversity of physiological or synthetic antigens is predominantly 
Table 1 Propensity of three classes of genes involved in different Alternative Splicing associated processes and their $Z$ - values of pair wise comparisons

\begin{tabular}{|c|c|c|c|c|c|c|c|c|c|c|c|}
\hline & \multicolumn{3}{|c|}{ Propensity of genes (\%) } & \multicolumn{5}{|c|}{ Propensity of genes (\%) } & \multicolumn{3}{|c|}{ Propensity of genes (\%) } \\
\hline & $A D$ & ID & IG & & $A D$ & ID & IG & & $A D$ & ID & IG \\
\hline $\begin{array}{l}\text { Alternative } \\
\text { Splicing }\end{array}$ & 79.5 & 84.38 & 89.79 & $\begin{array}{l}\text { NMD-linked } \\
\text { mRNA decay }\end{array}$ & 11.65 & 18.2 & 34 & $\begin{array}{l}\text { 5'splice } \\
\text { Site } \\
\text { SNPs }\end{array}$ & 23.77 & 14.58 & 8.73 \\
\hline $\begin{array}{c}\text { Significant } \\
\text { Level } \\
\text { (at } 95 \% \\
\text { confidence } \\
\text { level) }\end{array}$ & $\begin{array}{c}Z=2.6 \\
(A D \text { vs. ID) }\end{array}$ & $\begin{array}{c}Z=3.9 \\
(I D \text { vs IG) }\end{array}$ & $\begin{array}{c}Z=7.5 \\
\text { (IG vs. AD) }\end{array}$ & & $\begin{array}{c}Z=1.7 \\
(A D \text { vs. ID) }\end{array}$ & $\begin{array}{c}Z=5.5 \\
(I D \text { vs. IG) }\end{array}$ & $\begin{array}{c}Z=7.6 \\
(I G \text { vs. AD) }\end{array}$ & & $\begin{array}{c}Z=1.03 \\
(A D \text { vs. ID) }\end{array}$ & $\begin{array}{c}Z=4.8 \\
(I D \text { vs. IG) }\end{array}$ & $\begin{array}{c}Z=7.5 \\
\text { (IG Vs. AD) }\end{array}$ \\
\hline
\end{tabular}

rendered by intrinsically disordered regions of proteins [39]. Association with a large number of disorder residues of IG is also be an imperative reason for their faster evolutionary rate than AD and ID genes since in some protein families it has been demonstrated that the disordered regions evolve at a significantly faster rate than the ordered regions [40].

\section{Role of SNPs on Transcript level Variations}

In recent years there has been growing evidence for extensive natural variations like SNPs to be the major contributor of alternative splicing variation in humans [41]. Numerous disease-causing mutations within the consensus 5' splice site create a cryptic splice site that leads to defective mRNA and protein products $[42,43]$. In our study, we also noticed a greater association of 5', splice site SNPs (ss SNP) with AD genes compared to ID and IG (Table 1). This phenomenon indicates that SNPs impede the disease genes (AD, ID) to take part in alternative splicing by altering the splicing signals and their lower involvement with alternative splicing than IG genes may imposes much more evolutionary pressure on disease genes.

\section{Effects of Protein level Variations}

All proteins are potentially subjected to Post-translational Modifications (PMs) to accomplish many important roles in regulating the biological processes such as regulation of gene expression, activation/deactivation of enzymatic activity, protein stability or destruction, mediation of protein-protein interactions etc [44]. However, in some cases, PMs may be detrimental to protein functionality and may compromise the cellular functions in which they reside [45]. Among many of the modifications, post-translational phosphorylation is one of the most common protein modifications that occur in animal cells. Calculation of PMs sites revealed that the fraction of potential phosphorylation residues i.e serine, threonine, and cysteine to the total length of the protein is significantly (Mann-Whitney's $\mathrm{P}<0.05$ in each case) higher in case of AD genes (0.097) compared to ID
(0.084) and IG (0.069) genes. This observation again emphasized the previous hypothesis that the abnormal frequency of PMs uncover cryptic epitopes or create some novel epitopes that may be not tolerated during T-cell selection and trigger the pathogenesis of autoimmune disorder [45]. Contextually, it has recently been discovered that an additional purifying selection are operated on the positions involved in phosphorylation as compared to their unmodified counterparts in the same protein [46]. Thus the higher enrichment of posttranslational phosphorylation site in AD genes may be considered as a potential reason for their lower evolutionary rate.

Furthermore, it is well established that buried residues in a protein are important determinants of protein stability while surface residues are involved in protein function [47]. Here we found that AD genes bury more residue on average compared to ID and IG genes (Figure 1). Since buried residues evolve at a slower rate [48], the higher level of residue burial in AD genes can be accounted for their lower sequence divergence and as well as a possible means of achieving greater stability.

\section{Role of SNPs on Protein level Variations}

Systematic approach to the analysis of SNPs indicated that SNPs resulting in deleterious amino acid changes predominantly affect the stability of the protein [49]. We then map the non-synonymous SNPs on protein buried region and quantify the hydrophobic, hydrophilic, amphipathic amino acid substitution frequency in each group of genes. The average amino acid exchange frequencies among hydrophobic, hydrophilic and amphipathic amino acids among AD, ID, IG genes for buried regions of proteins are diagrammatically represented in Figure 2. We noticed transition from hydrophilic to hydrophobic or amphipathic to hydrophobic residue is more frequently substituted in the buried regions of $\mathrm{AD}$ proteins compared to ID and IG proteins. Moreover, the hydrophobicity of buried region in $\mathrm{AD}$ genes has found to increase significantly after substitution with SNPs than ID genes while no change of hydrophobicity has 


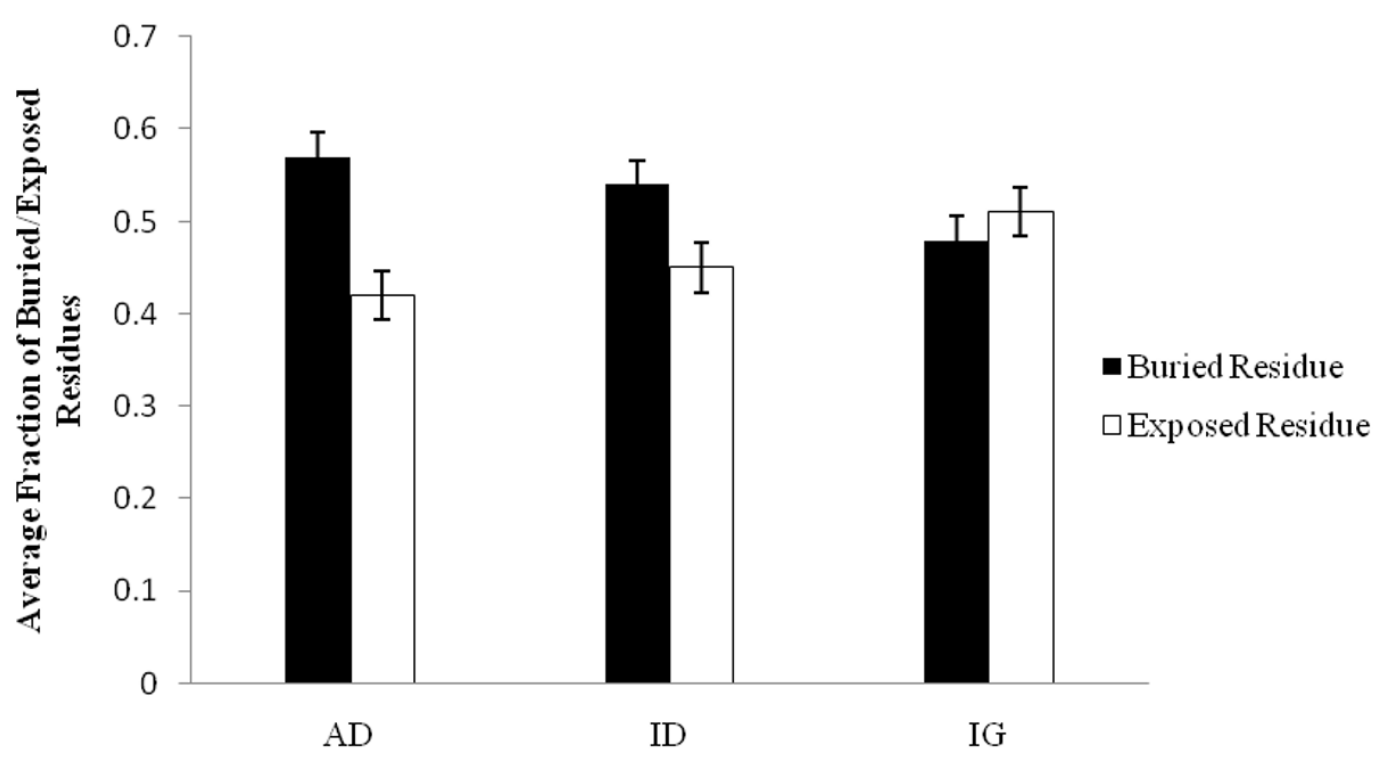

Figure 1 Distributions of buried and exposed residues among AD, ID and IG proteins. Error bar represents $5 \%$ standard error of data.

occurred in case of IG (Figure 3). Thus, influence of SNPs in increasing the hydrophobicity in buried region of AD proteins may be responsible for evolutionary constraint for maintaining protein stability.

\section{Relative Contribution of the Factors in Determining Evolutionary Rate Variation}

Here, we noticed different probable factors in the three levels (DNA-m-RNA-Protein) that can explain evolutionary rate differences among $\mathrm{AD}$, ID and IG genes. To assess the contribution of each variable, we compute Principal Component Analysis (PCA). The dominant eigen vectors (taken as equal to or greater than 1) that appear from this analysis can be interpreted as the most important contributors directing protein evolution [50]. PCA with gene level variables (SNPs, CNVs, RR, duplicability); m-RNA level variables (isoform number, alternatively spliced exon, m-RNA abundance, disorderness); protein level variables (phosphorylation, protein residue burial), which are the dominant factors, are represented in table 2. Multiple Regression Analysis was then performed to assess the contribution of each level variables determined in PCA in a single regression model from which we can identify the influence of all potential

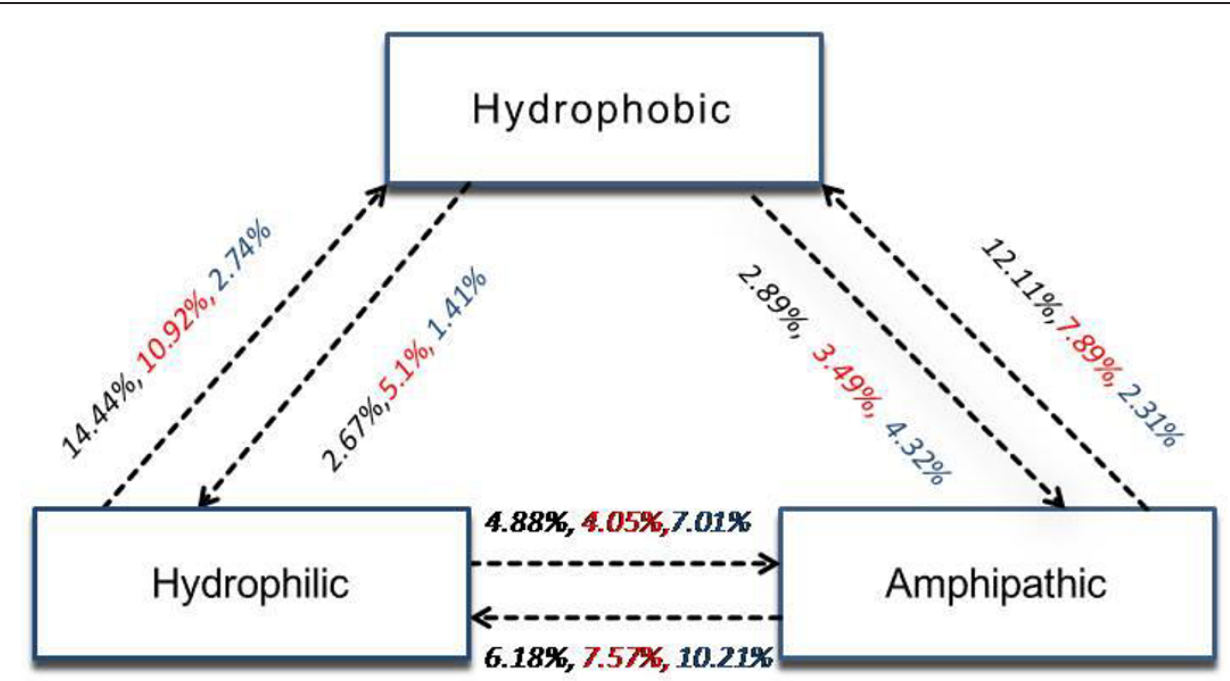

Figure 2 Average amino acid exchange frequencies due to Single Nucleotide Polymorphisms (SNPs) among hydrophobic, amphipathic and hydrophilic amino acids for AD (black), ID (red), IG (blue) proteins. 


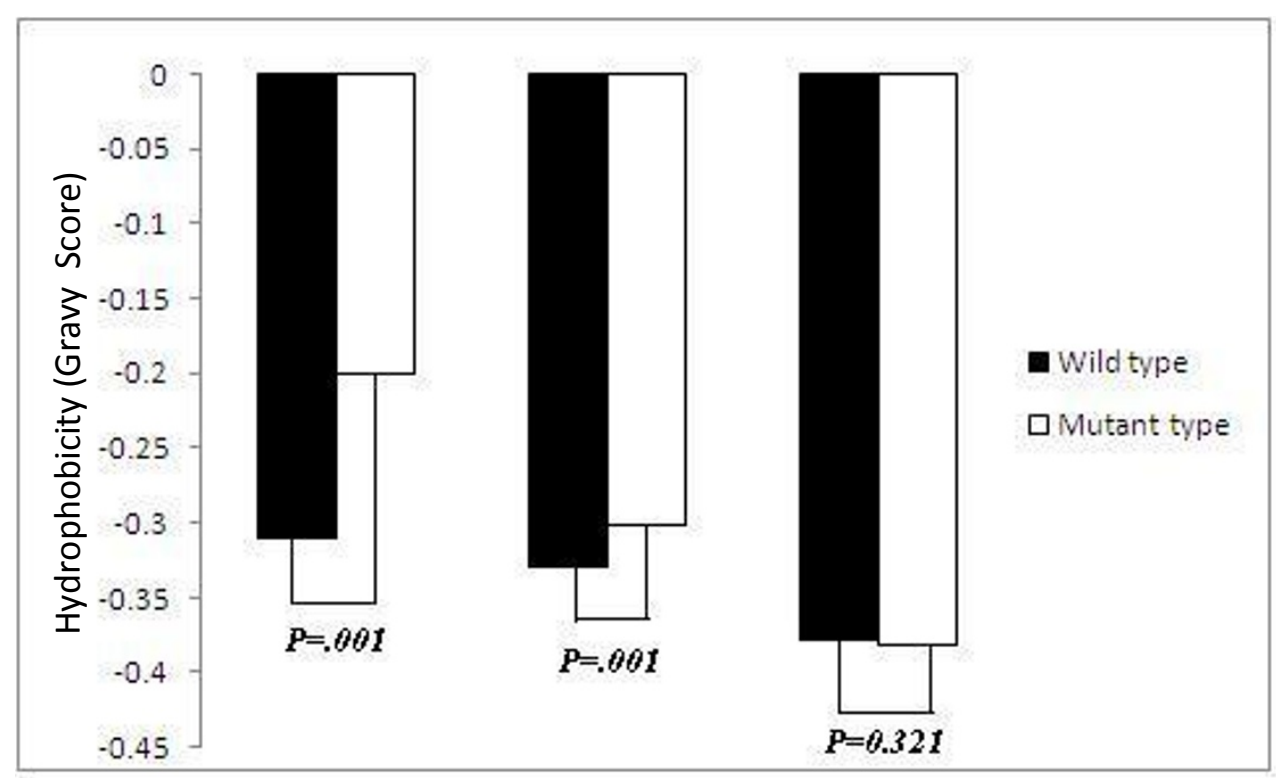

Figure 3 Differences in average hydrophobicity score between the three categories of genes before mutation (wild type) and after mutation (mutant type) with SNPs. P-value shows the significant level.

predictor variables and at the same time can eliminate step by step those predictors that contribute least to the regression model. Regression analysis exhaustively confirmed that the SNPs $(\beta=-3.725)$, is the most influential predictor of the evolutionary rate followed by the $\mathrm{m}$ RNA abundance $(\beta=-3.005)$, paralogs number $(\beta=$ -2.036), fraction of phosphorylation residue $(\beta=-2.091)$, alternatively spliced exon $(\beta=1.960)$, protein residue burial $(\beta=-1.085)$ and protein disorder $(\beta=1.021)$.

\section{Discussion}

Recent years have witnessed rapid progress in elucidating the molecular causes of various diseases. Here we analyzed the evolutionary disparity between the functional and non-functional immune systems. We noticed that autoimmune disease genes are more conserved than other immunological disease genes and both sets of genes evolved significantly at a slower rate than immunological genes. Though the evolutionary rates differences among the gene groups are statistically significant, the difference of mean values between autoimmune and immunological disease genes is small. However, the differences of mean values among the groups turned out to be prominent when we analyzed non-synonymous and synonymous substitution rates separately $(\mathrm{dn}$ for autoimmune disease genes $=0.0079$; immunological disease genes $=0.0091$; immunological genes $=0.0118$; Mann-Whitney's $\mathrm{P}=1 \times 10^{-3}$ in each case and ds for autoimmune disease genes $=0.0232$; immunological disease genes $=0.0254$; immunological genes $=0.0291$; Mann-Whitney's $\mathrm{P}<0.05$ in each case).
Significant differences in synonymous substitutions rates among the gene groups indicate the role of neutral substitutions in driving the evolutionary rate discrepancies among them. Now, the slower evolving disease linked immune genes raise a fundamental question why non disease immune genes evolve at a higher rate compared to disease related immune genes since it was previously documented by several studies that non-disease genes evolve at a slower rate than disease genes [51,52], though some controversial reports [11] are also present. To resolve this controversy, our previous study [12] exemplified that, monogenic diseases inherited by Mendelian fashion and polygenic disease genes inherited by non-Mendelian fashion are evolutionarily faster than housekeeping genes but monogenic disease genes show slower evolutionary rate than tissue specific genes. It is also noteworthy to mention that immune system genes show tissue-specific expression pattern [53] and both of our disease datasets mostly comprise monogenic disease genes (autoimmune disease genes dataset: 69\% monogenic disorder, $31 \%$ polygenic disorder; other immunological disease genes dataset: $61 \%$ monogenic disorder; $39 \%$ polygenic disorder). Herein, the differences in single nucleotide polymorphisms, copy number variations, recombination rate, duplicability, alternative splicing, disorderness, post-translational modification, and protein residue burial can explain the evolutionary disparity among the three groups of genes.

The evolutionary conservation of disease related immunological genes in spite of their higher association with non-synonymous single nucleotide polymorphisms 
Table 2 Principal Component Analysis on Evolutionary Rate ( $\omega$ ) with (a) Gene Level Predictors, (b) Transcript Level Predictors, (c) Protein Level Predictors.

\begin{tabular}{|c|c|c|}
\hline \multicolumn{3}{|l|}{ a. Gene Level Predictors } \\
\hline & Principal Component1 & Principal Component2 \\
\hline $\begin{array}{l}\text { Percent of the total variance } \\
\text { Correlation Coefficient (Spearman's } \rho \text { ) with } \omega\end{array}$ & $\begin{array}{l}27.502 \\
-0.061^{* *}\end{array}$ & $\begin{array}{l}21.912 \\
0.113^{* *}\end{array}$ \\
\hline \multicolumn{3}{|l|}{ Major Contributing Factor in PCA1 } \\
\hline $\begin{array}{l}\text { Paralogs Number } \\
\text { Recombination Rate }\end{array}$ & $\begin{array}{l}0.789 \\
0.805\end{array}$ & - \\
\hline \multicolumn{3}{|l|}{ Major Contributing Factor in PCA2 } \\
\hline $\begin{array}{l}\text { Single Nucleotide Polymorphism } \\
\text { Copy Number Variation }\end{array}$ & $\overline{-}$ & $\begin{array}{l}0.995 \\
0.713\end{array}$ \\
\hline \multicolumn{3}{|l|}{ a. Transcript Level Predictors } \\
\hline $\begin{array}{l}\text { Percent of the total variance } \\
\text { Correlation Coefficient (Spearman's } \rho \text { ) with } \omega\end{array}$ & $\begin{array}{l}39.071 \\
-0.161^{* *}\end{array}$ & \\
\hline \multicolumn{3}{|l|}{ Major Contributing Factor in PCA1 } \\
\hline $\begin{array}{l}\text { m-RNA abundance } \\
\text { Alternatively Spliced Exons } \\
\text { Disorderness }\end{array}$ & $\begin{array}{l}0.702 \\
0 \\
0.688 \\
0.446\end{array}$ & \\
\hline \multicolumn{3}{|l|}{ c.Protein Level Predictors } \\
\hline $\begin{array}{l}\text { Percent of the total variance } \\
\text { Correlation Coefficient (Spearman's } \rho \text { ) with } \omega\end{array}$ & $\begin{array}{l}69.095 \\
-0.321^{* * *}\end{array}$ & \\
\hline \multicolumn{3}{|l|}{ Major Contributing Factor in PCA1 } \\
\hline $\begin{array}{l}\text { Fraction of Phosphorylation Residues } \\
\text { Proportion of Buried Residues }\end{array}$ & $\begin{array}{l}0.557 \\
0.743\end{array}$ & \\
\hline
\end{tabular}

N.B.: * denotes $P<10^{-3}$; ** denotes $P<10^{-6} ; * *$ denotes $P<10^{-9}$.

is an artifact of its beneficial impact on disease related genes (Figure 4). Single nucleotide polymorphisms upregulate recombination rates which in turns increase the gene expression as well as paralogs number in disease genes. Duplication driven disease gene formation has also supported by a series of evidence in an earlier literature [54]. Previously, it was underscored that duplication and alternative splicing could not be operated simultaneously rather they hold a negative correlation with each other [55]. Since the disease genes achieved their proteome size through gene duplication, we observed a lower involvement with alternative splicing. Here also single nucleotide polymorphisms played a critical role in 5 ' splice site and create a cryptic splice site by altering the splicing signal. On the other hand the immunological genes follow the path of alternative splicing to enhance their diversity. However, the frequent link with alternative splicing could not generate higher m-RNA abundance of immunological genes due to "Regulated Unproductive Splicing and Translation" (RUST) mechanism [56] in which premature termination codon containing isoforms are targeted to nonsense mediated decay to regulate the transcript level of functional protein. Rather alternative splicing helps to impose a greater flexibility to bind with an enormous number of foreign particles without known structural analogy through increasing protein disorderness in immunological genes (Spearman's $\rho$ disorderness, alternatively spliced exon=0.134, $\left.\mathrm{P}=1 \times 10^{-3}\right)$. Thus, we deciphered that the basic difference in the involvement of proteome expansion machinery put differential selective pressures on malfunctioning immune genes and the functional immune genes. Moreover, it is also observed in our search that autoimmune disease and other immunological disease genes are more prone to post-translational phosphorylation which may regarded as a possible reason for slower evolutionary rate. In protein structure level, the higher residue burial is observed in two types of disease genes compared to non-disease genes and the propensity of single nucleotide polymorphisms to substitute hydrophilic, amphipathic amino acid by hydrophobic amino acid in disease groups could be prompted as a reason of lower sequence divergence in autoimmune disease and other immunological disease genes than immunological genes. Conferring structural stability to the autoimmune disease genes also has a biological significance since incidence of autoimmunity sharply increases in the stable protein forms in the cell [57].

\section{Conclusions}

Assessing the results from multivariate regression analysis we conclude that the relative dominance of individual factors modulating the differential substitution rate experienced by autoimmune disease, other immunological 


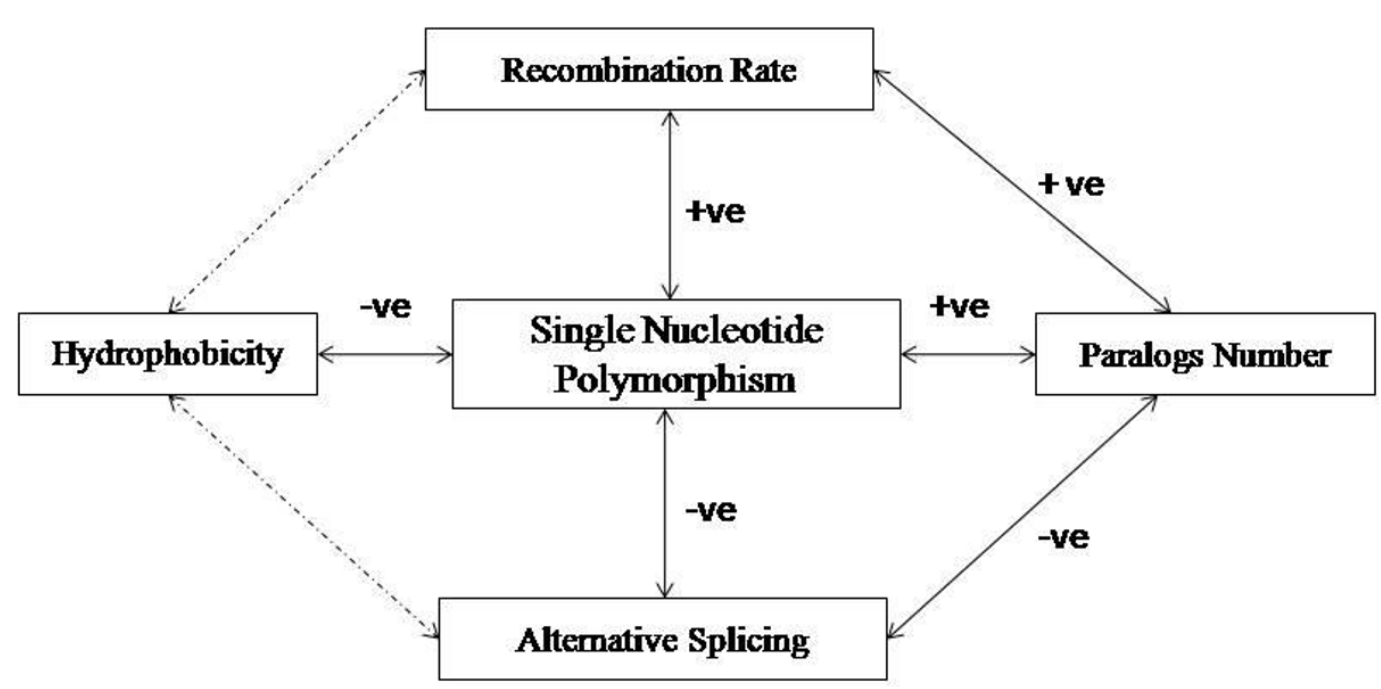

Figure 4 The schematic representation to illustrate synchronous effect of Single Nucleotide Polymorphisms on recombination rate, hydrophobicity, paralogs number, alternative splicing. Bold arrows denote significant correlations, +ve for positive and -ve for negative while the dotted arrow indicates non-significant (NS) relationship between variables.

disease and immunological disease genes is in the order of single nucleotide polymorphisms $>$ m-RNA abundance $>$ paralogs number $>$ phosphorylation residue $>$ alternatively spliced exon $>$ protein residue burial $>$ protein disorder. To the best of our knowledge, this is the first extensive comparison of disease and non-disease related immunological genes from evolutionary perspective. This finding also shades light into the mutational spectrum acting on DNAmRNA-protein level of the three classes of genes. Our study will surely enrich the knowledge of disease gene identification and may also help in medicinal improvement of autoimmune disease.

\section{Methods}

Immune Related Disease and Non-disease Genes Identification

Immune related disease genes mainly consist with Autoimmune disease, Immunoproliferative disease, Immunologic deficiency syndromes, hypersensitivity, Graft rejection, Purpura, thrombocytopenia, and Glomerulonephritis. There exists a clear demarcation between the basic disease mechanism of autoimmune disease and rest of the immune related disease genes. Thus immune related disease genes are broadly categorized into the two groups autoimmune disease genes and other immunological disease genes. These two types of genes inherited by Mendelian and non-Mendelian fashion were downloaded from Biobase and Genetic Association Database [58] respectively. Autoimmune disease genes include Rheumatoid Arthritis, Diabetes Mellitus, Systemic Lupus Erythematosus, Greves disease, Thyroiditis, Antiphospholipid Syndrom, Pemphigus, Polyendocrynopathis, Hemolytic anemia, Multiple Sclerosis etc. Then we have checked the functional description of the gene sets downloaded from Biobase and Genetic Association database. The link between the functional description and disease association was manually verified and the genes whose functional descriptions match with disease associations were considered in our study while the genes which are common in both autoimmune disease and other immunological diseases were excluded from our dataset. Since the main objective of our study is to find out the evolutionary disparity among the gene sets, we have chosen only those genes for which the information is available for their orthologs in Chimpanzee and their $\mathrm{dn}$ and ds values in Ensembl. Finally we have constructed the dataset with a total of 781 autoimmune disease genes and 679 other immunological disease genes (Additional file 1, Table S1). Immunological genes were obtained from ImmPort [59] and filtered with similar criteria. Finally we have acquired 2470 non-disease immunological genes by excluding the above disease genes list (Additional file 1, Table S1).

\section{Orthologs and Paralogs Identification}

The gene sequences, paralogs information, pair-wise non-synonymous substitution rates $(\mathrm{dn})$ and synonymous substitution rates (ds) with Chimp (1:1) orthologs corresponding to both types of immunological disease genes as well as non-disease genes were retrieved from Ensembl [60].

\section{Gene Expression Profile}

The gene expression profile data was extracted from BioGPS dataset [61]. The signal intensities across 79 
tissues were averaged and were considered as expression level for each gene represented by their corresponding probe id. mRNA abundance of the genes in our dataset was calculated using EST data obtained from DFCI Gene Indices. Gene expression level was estimated by calculating the number of occurrences of each gene among EST sequences from 179 cDNA libraries sampled with at least 10,000 ESTs [62]. Eliminating pathogenic and cancerous libraries, 41 libraries were kept and alignments were made between the coding sequences of the gene groups with the EST dataset using BLASTN program with a sequence matching criterion of $60 \%$ identity and $80 \%$ overlaps. The overall EST counts for each gene across 41 EST libraries represented their mRNA abundance.

\section{Measurement of SNPs, CNVs, Recombination Rate}

Non-synonymous SNPs and CNVs information were downloaded from Polydoms [63] and Database of Genomic Variants [64] respectively. Chromosome wise gene recombination rates were downloaded from Hapmap project [65]. The recombination rates of the progenitor genes were calculated using the formula $\Sigma \rho_{\mathrm{i}} / \mathrm{l}$, where $\rho_{\mathrm{i}}$ stands for recombination rate at a base position and 1 for the genic length corresponding to that gene [66].

\section{Alternative Splicing and SNPs Effect}

Data for alternatively spliced isoforms and exons for the genes in the dataset were downloaded from the Alternative Splicing Annotation Project [67]. Splice site SNPs information were collected from ssSNP Target [68]. Data for alternatively spliced isoforms that are coupled to mRNA degradation were fetched from AS-ALPS [69].

\section{Prediction of Intrinsically Disorder Region, Hydrophobicity and Post-translational Phosphorylation}

Disorder predictions were carried out using the program FoldIndex [70] implementing the prediction method of Uversky et al. [71]. Post translational phosphorylation in the disease and non-disease related immunological proteins were measured from NetPhos (2.0) [72]. Hydrophobicity values of proteins were retrieved from ProtParam [73]

\section{Calculation of Buried and Exposed Residues in Protein}

Residue-wise burial in proteins was computed by a standalone version of sequence-based prediction program RVP-Net [74]. This program relies on a neural network trained to estimate solvent accessibility of each residue from sequence features and was trained over non-redundant set of protein structures. Predicted relative solvent accessible surface area was converted to a two burial classes (buried/exposed) at 16\% cutoff, which roughly corresponds to the median of solvent accessibility distribution in training proteins. The classification of amino acids as hydrophobic, hydrophilic and amphipathic were done according to ref [75].

\section{Statistical Test}

We performed Mann-Whitney U test for pair-wise comparisons since the values are not normally distributed in our dataset. Multiple regression analysis, Principal component analysis were performed for relative contribution analysis of each factors to evolutionary rate. All the statistical tests were carried out by the SPSS (13.0) package.

\section{Additional material}

Additional file 1: Supplementary Table S1. Genomic and proteomic features of Autoimmune disease, Immunological disease gens and Immunological genes.

\section{Abbreviations}

AD: Autoimmune Disease; ID: Immunological Disease; IG: Immunological Genes; RR: Recombination Rate; AS: Alternative Splicing; NMD: Non-sense Mediated mRNA Decay; PMs: Posttranslational Modifications; ssSNP: splice site SNPs; PCA: Principal Component Analysis; PTC: Premature Termination Codon.

\section{Acknowledgements}

We thank Professor S. Ahmad for calculation protein accessible surface area (ASA). Work has done by financial help of Department of Biotechnology, Govt. of India. We thank to two anonymous referees for their constructing remarks in improving the manuscript.

\section{Authors' contributions}

SP made the analyses and drafted the manuscript. TCG guided the work and helped to draft the manuscript. All authors read and approved the final manuscript.

\section{Competing interests}

The authors declare that they have no competing interests.

Received: 29 September 2011 Accepted: 25 January 2012 Published: 25 January 2012

\section{References}

1. Hughes AL, Packer B, Welch R, Chanock SJ, Yeager M: High level of functional polymorphism indicates a unique role of natural selection at human immune system loci. Immunogenetics 2005, 57:821-827.

2. Park SG, Choi SS: Expression breadth and expression abundance behave differently in correlations with evolutionary rates. BMC Evol Biol 2010, $10: 241$.

3. Limaye N, Belobrajdic KA, Wandstrat AE, Bonhomme F, Edwards SV, Wakeland EK: Prevalence and evolutionary origins of autoimmune susceptibility alleles in natural mouse populations. Genes Immun 2008 9:61-68.

4. Hahn MW, Demuth JP, Han SG: Accelerated rate of gene gain and loss in primates. Genetics 2007, 177:1941-1949.

5. Bustamante CD, Fledel-Alon A, Williamson S, Nielsen R, Hubisz MT, Glanowski S, Tanenbaum DM, White TJ, Sninsky JJ, Hernandez RD, et al: Natural selection on protein-coding genes in the human genome. Nature 2005, 437:1153-1157.

6. Thomas JK, Richard AG, Barbara AO, Janis K: Kuby Immunology. 6 edition. W. h.freeman \& Co Ltd; 2006.

7. Henderson RD, Bain CJ, Pender MP: The occurrence of autoimmune diseases in patients with multiple sclerosis and their families. J Clin Neurosci 2000, 7:434-437. 
8. Aune TM, Parker JS, Maas K, Liu Z, Olsen NJ, Moore JH: Co-localization of differentially expressed genes and shared susceptibility loci in human autoimmunity. Genet Epidemiol 2004, 27:162-172.

9. Wegner N, Wait R, Venables PJ: Evolutionarily conserved antigens in autoimmune disease: implications for an infective aetiology. Int J Biochem Cell Biol 2009, 41:390-397.

10. Ng B, Yang F, Huston DP, Yan Y, Yang Y, Xiong Z, Peterson LE, Wang H, Yang XF: Increased noncanonical splicing of autoantigen transcripts provides the structural basis for expression of untolerized epitopes. $J$ Allergy Clin Immunol 2004, 114:1463-1470.

11. Huang $\mathrm{H}$, Winter EE, Wang $\mathrm{H}$, Weinstock KG, Xing H, Goodstadt L, Stenson PD, Cooper DN, Smith D, Albà MM, et al: Evolutionary conservation and selection of human disease gene orthologs in the rat and mouse genomes. Genome Biol 2004, 5:R47.

12. Podder $\mathrm{S}$, Ghosh TC: Exploring the differences in evolutionary rates between monogenic and polygenic disease genes in human. Mol Biol Evol 2010, 27:934-941.

13. Podder S, Ghosh TC: Insights into the molecular correlates modulating functional compensation between monogenic and polygenic disease gene duplicates in human. Genomics 2011, 97:200-204.

14. Sabeti PC, Varilly P, Fry B, Lohmueller J, Hostetter E, Cotsapas C, Xie X, Byrne EH, McCarroll SA, Gaudet R, et al: Genome-wide detection and characterization of positive selection in human populations. Nature 2007, 449:913-918

15. Chen FC, Wang SS, Chen CJ, Li WH, Chuang TJ: Alternatively and constitutively spliced exons are subject to different evolutionary forces. Mol Biol Evol 2006, 23:675-682.

16. Stephan W, Langley CH: Molecular genetic variation in the centromeric region of the $\mathrm{X}$ chromosome in three Drosophila ananassae populations. I. Contrasts between the vermilion and forked loci. Genetics 1989, 121:89-99.

17. Begun DJ, Aquadro CF: Molecular population genetics of the distal portion of the $\mathrm{X}$ chromosome in Drosophila: evidence for genetic hitchhiking of the yellow-achaete region. Genetics 1991, 129:1147-1158.

18. Nachman MW, Bauer VL, Crowell SL, Aquadro CF: DNA variability and recombination rates at X-linked loci in humans. Genetics 1998, 150:1133-1141

19. Dvorák J, Luo MC, Yang ZL: Restriction fragment length polymorphism and divergence in the genomic regions of high and low recombination in self-fertilizing and cross-fertilizing aegilops species. Genetics 1998, 148:423-434.

20. Hill WG, Robertson A: The effect of linkage on limits to artificial selection. Genet Res 1966, 8:269-294.

21. Connallon T, Knowles LL: Recombination rate and protein evolution in yeast. BMC Evol Biol 2007, 7:235.

22. Pál C, Papp B, Hurst LD: Does the recombination rate affect the efficiency of purifying selection? The yeast genome provides a partial answer. Mol Biol Evol 2001, 18:2323-2326.

23. Zhang L, Lu HH, Chung WY, Yang J, Li WH: Patterns of segmental duplication in the human genome. Mol Biol Evol 2005, 22:135-141.

24. Sen K, Podder S, Ghosh TC: Insights into the genomic features and evolutionary impact of the genes configuring duplicated pseudogenes in human. FEBS Lett 2010, 584:4015-4018.

25. Jordan IK, Wolf YI, Koonin EV: Duplicated genes evolve slower than singletons despite the initial rate increase. BMC Evol Biol 2004, 4:22.

26. Hastings PJ, Lupski JR, Rosenberg SM, Ira G: Mechanisms of change in gene copy number. Nat Rev Genet 2009, 10:551-564.

27. Nguyen DQ, Webber C, Hehir-Kwa J, Pfundt R, Veltman J, Ponting CP: Reduced purifying selection prevails over positive selection in human copy number variant evolution. Genome Res 2008, 18:1711-1723.

28. Nguyen DQ, Webber C, Ponting CP: Bias of selection on human copynumber variants. PLOS Genet 2006, 2:e20.

29. Modrek B, Lee C: A genomic view of alternative splicing. Nat Genet 2002, 30:13-19.

30. Ramensky VE, Nurtdinov RN, Neverov AD, Mironov AA, Gelfand MS: Positive selection in alternatively spliced exons of human genes. Am J Hum Genet 2008, 83:94-98

31. Chen FC, Wang SS, Chen CJ, Li WH, Chuang TJ: Alternatively and constitutively spliced exons are subject to different evolutionary forces. Mol Biol Evol 2006, 23:675-682.
32. Lynch KW: Consequences of regulated pre-mRNA splicing in the immune system. Nat Rev Immunol 2004, 4:931-940.

33. Zhang H, Wang L, Song L, Zhao J, Qiu L, Gao Y, Song X, Li L, Zhang Y, Zhang $L$ : The genomic structure, alternative splicing and immune response of Chlamys farreri thioester-containing protein. Dev Comp Immunol 2009, 33:1070-1076.

34. Lewis BP, Green RE, Brenner SE: Evidence for the widespread coupling of alternative splicing and nonsense-mediated mRNA decay in humans. Proc Natl Acad Sci USA 2003, 100:189-192.

35. McGlincy NJ, Smith CW: Alternative splicing resulting in nonsensemediated mRNA decay: what is the meaning of nonsense? Trends Biochem Sci 2008, 33:385-393.

36. Edwards YJ, Lobley AE, Pentony MM, Jones DT: Insights into the regulation of intrinsically disordered proteins in the human proteome by analyzing sequence and gene expression data. Genome Biol 2009, 10:R50.

37. Romero PR, Zaidi S, Fang YY, Uversky VN, Radivojac P, Oldfield CJ, Cortese MS, Sickmeier M, LeGall T, Obradovic Z, Dunker AK: Alternative splicing in concert with protein intrinsic disorder enables increased functional diversity in multicellular organisms. Proc Natl Acad Sci USA 2006, 103:8390-8395

38. Kovacs E, Tompa P, Liliom K, Kalmar L: Dual coding in alternative reading frames correlates with intrinsic protein disorder. Proc Natl Acad Sci USA 2010, 107:5429-5434

39. Uversky VN, Oldfield CJ, Dunker AK: Showing your ID: intrinsic disorder as an ID for recognition, regulation and cell signaling. J Mol Recognit 2005, 18:343-384.

40. Brown CJ, Takayama S, Campen AM, Vise P, Marshall TW, Oldfield CJ, Williams CJ, Dunker AK: Evolutionary rate heterogeneity in proteins with long disordered regions. J Mol Evol 2002, 55:104-110.

41. Montgomery SB, Sammeth M, Gutierrez-Arcelus M, Lach RP, Ingle C, Nisbett J, Guigo R, Dermitzakis ET: Transcriptome genetics using second generation sequencing in a Caucasian population. Nature 2010, 464:773-777.

42. Krawczak M, Reiss J, Cooper DN: The mutational spectrum of single basepair substitutions in mRNA splice junctions of human genes: causes and consequences. Hum Genet 1992, 90:41-54.

43. Krawczak M, Thomas NS, Hundrieser B, Mort M, Wittig M, Hampe J, Cooper DN: Single base-pair substitutions in exon-intron junctions of human genes: nature, distribution, and consequences for mRNA splicing. Hum Mutat 2007, 28:150-158.

44. Walsh CT: Posttranslational modification of proteins: expanding nature's inventory. Englewood, CO: Roberts and Company Publishers; 2006.

45. Cloos PA, Christgau S: Post-translational modifications of proteins: implications for aging, antigen recognition, and autoimmunity. Biogerontology 2004, 5:139-158.

46. Gray VE, Kumar S: Rampant purifying selection conserves positions with posttranslational modifications in human proteins. Mol Biol Evol 2011, 28:1565-1568.

47. Ponder JW, Richards FM: Tertiary templates for proteins. Use of packing criteria in the enumeration of allowed sequences for different structural classes. J Mol Biol 1987, 193:775-791.

48. Goldman N, Thorne JL, Jones DT: Assessing the impact of secondary structure and solvent accessibility on protein evolution. Genetics 1998 149:445-458.

49. Sunyaev S, Ramensky V, Koch I, Lathe W, Kondrashov AS, Bork P: Prediction of deleterious human alleles. Hum Mol Genet 2001, 10:591-597.

50. Chakraborty S, Kahali B, Ghosh TC: Protein complex forming ability is favored over the features of interacting partners in determining the evolutionary rates of proteins in the yeast protein-protein interaction networks. BMC Syst Biol 2010, 4:155.

51. Smith NG, Eyre-Walker A: Human disease genes: patterns and predictions. Gene 2003, 318:169-175.

52. López-Bigas N, Ouzounis CA: Genome-wide identification of genes likely to be involved in human genetic disease. Nucleic Acids Res 2004, 32:3108-3114.

53. Greco D, Somervuo P, Di Lieto A, Raitila T, Nitsch L, Castrén E, Auvinen P: Physiology, pathology and relatedness of human tissues from gene expression meta-analysis. PLoS One 2008, 3:e1880.

54. Conrad B, Antonarakis SE: Gene duplication: a drive for phenotypic diversity and cause of human disease. Annu Rev Genomics Hum Genet 2007, 8:17-35. 
55. Su Z, Wang J, Yu J, Huang X, Gu X: Evolution of alternative splicing after gene duplication. Genome Res 2006, 16:182-189.

56. Cuccurese M, Russo G, Russo A, Pietropaolo C: Alternative splicing and nonsense-mediated mRNA decay regulate mammalian ribosomal gene expression. Nucleic Acids Res 2005, 33:5965-5977.

57. Khadra A, Santamaria P, Edelstein-Keshet L: The pathogenicity of selfantigen decreases at high levels of autoantigenicity: a computational approach. Int Immunol 2010, 22:571-582.

58. Becker KG, Barnes KC, Bright TJ, Wang SA: The genetic association database. Nat Genet 2004, 36:431-432

59. Collison LW, Chaturvedi V, Henderson AL, Giacomin PR, Guy C, Bankoti J, Finkelstein D, Forbes K, Workman CJ, Brown SA, et al: IL-35-mediated induction of a potent regulatory T cell population. Nat Immunol 2010, 11:1093-1101.

60. Flicek P, Amode MR, Barrell D, Beal K, Brent S, Chen Y, Clapham P, Coates G, Fairley S, Fitzgerald S, et al: Ensembl 2011. Nucleic Acids Res 2011, 39: D800-806.

61. Su Al, Wiltshire T, Batalov S, Lapp H, Ching KA, Block D, Zhang J, Soden R, Hayakawa M, Kreiman $G$, et al: A gene atlas of the mouse and human protein-encoding transcriptomes. Proc Natl Acad Sci USA 2004, 101:6062-6067.

62. Podder S, Mukhopadhyay P, Ghosh TC: Multifunctionality dominantly determines the rate of human housekeeping and tissue specific interacting protein evolution. Gene 2009, 439:11-16.

63. Jegga AG, Gowrisankar S, Chen J, Aronow BJ: PolyDoms: a whole genome database for the identification of non-synonymous coding SNPs with the potential to impact disease. Nucleic Acids Res 2007, 35:D700-706.

64. Zhang J, Feuk L, Duggan GE, Khaja R, Scherer SW: Development of bioinformatics resources for display and analysis of copy number and other structural variants in the human genome. Cytogenet Genome Res 2006, 115:205-214

65. Thorisson GA, Smith AV, Krishnan L, Stein LD: The International HapMap Project Web site. Genome Res 2005, 15:1592-1593.

66. Kato M, Miya F, Kanemura Y, Tanaka T, Nakamura Y, Tsunoda T: Recombination rates of genes expressed in human tissues. Hum $\mathrm{Mol}$ Genet 2008, 17:577-586.

67. Lee C, Atanelov L, Modrek B, Xing Y: ASAP: the Alternative Splicing Annotation Project. Nucleic Acids Res 2003, 31:101-105.

68. Yang JO, Kim WY, Bhak J: ssSNPTarget: genome-wide splice-site Single Nucleotide Polymorphism database. Hum Mutat 2009, 30:E1010-1020.

69. Shionyu M, Yamaguchi A, Shinoda K, Takahashi K, Go M: AS-ALPS: a database for analyzing the effects of alternative splicing on protein structure, interaction and network in human and mouse. Nucleic Acids Res 2009, 37:D305-309.

70. Prilusky J, Felder CE, Zeev-Ben-Mordehai T, Rydberg EH, Man O, Beckmann JS, Silman I, Sussman JL: Foldindex: a simple tool to predict whether a given protein sequence is intrinsically unfolded. Bioinformatics 2005, 21:3435-3438.

71. Uversky VN, Gillespie JR, Fink AL: Why are "natively unfolded" proteins unstructured under physiologic conditions? Proteins 2000, 41:415-427.

72. Blom N, Gammeltoft S, Brunak S: Sequence and structure-based prediction of eukaryotic protein phosphorylation sites. J Mol Biol 1999, 294:1351-1362.

73. Gasteiger E, Gattiker A, Hoogland C, Ivanyi I, Appel RD, Bairoch A: ExPASy: The proteomics server for in-depth protein knowledge and analysis. Nucleic Acids Res 2003, 31:3784-3788.

74. Ahmad S, Gromiha MM, Sarai A: RVP-net: online prediction of real valued accessible surface area of proteins from single sequences. Bioinformatics 2003, 19:1849-1851.

75. D'Onofrio G, Jabbari K, Musto H, Bernardi G: The correlation of protein hydropathy with the base composition of coding sequences. Gene 1999, 238:3-14.

doi:10.1186/1471-2148-12-10

Cite this article as: Podder and Ghosh: Evolutionary dynamics of human autoimmune disease genes and malfunctioned immunological genes. BMC Evolutionary Biology 2012 12:10.

\section{Submit your next manuscript to BioMed Central and take full advantage of:}

- Convenient online submission

- Thorough peer review

- No space constraints or color figure charges

- Immediate publication on acceptance

- Inclusion in PubMed, CAS, Scopus and Google Scholar

- Research which is freely available for redistribution

Submit your manuscript at www.biomedcentral.com/submit 